\title{
Comparative analysis of four Zantedeschia chloroplast genomes: expansion and contraction of the IR region, phylogenetic analyses and SSR genetic diversity assessment
}

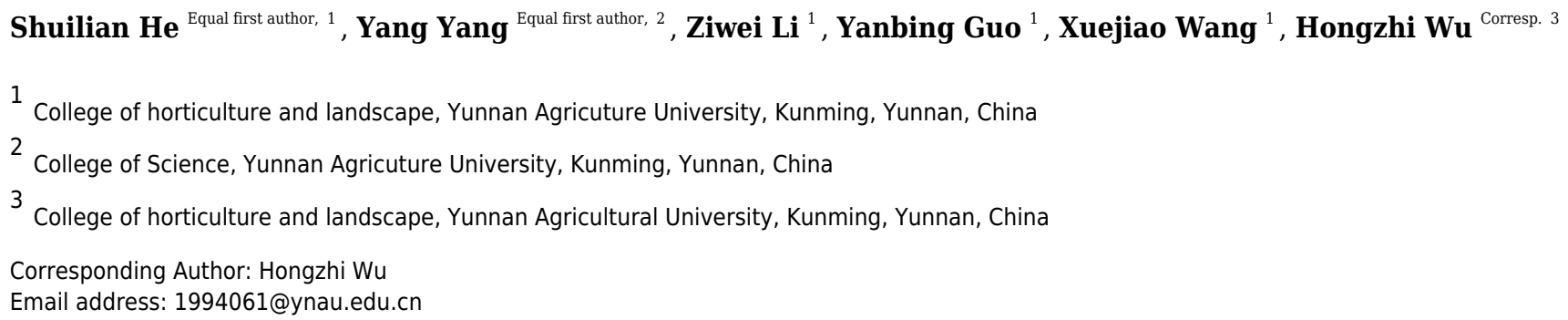

The horticulturally important genus Zantedeschia (Araceae) comprises eight species of herbaceous perennials. We sequenced, assembled and analyzed the chloroplast (cp) genomes of four species of Zantedeschia (Z. aethiopica, Z. odorata, Z. elliottiana, and Z. rehmannii) to investigate the structure of the $\mathrm{cp}$ genome in the genus. According to our results, the cp genome of Zantedeschia ranges in size from 169,065 bp ( $Z$. aethiopica) to $175,906 \mathrm{bp}$ (Z. elliottiana). We identified a total of 112 unique genes, including 78 proteincoding genes, 30 transfer RNA (tRNA) genes and four ribosomal RNA (rRNA) genes. Comparison of our results with cp genomes from other species in the Araceae suggests that the relatively large sizes of the Zantedeschia cp genomes may result from inverted repeats (IR) region expansion. The sampled Zantedeschia species formed a monophylogenetic clade in our phylogenetic analysis. Furthermore, the long single copy (LSC) and short single copy (SSC) regions in Zantedeschia are more divergent than the IR regions in the same genus, and non-coding regions showed generally higher divergence than coding regions. We identified a total of $410 \mathrm{cPSSR}$ sites from the four Zantedeschia species studied. Genetic diversity analyses based on four polymorphic SSR markers from 134 cultivars of Zantedeschia suggested that high genetic diversity $(I=0.934 ; \mathrm{Ne}=2.371$ ) is present in the Zantedeschia cultivars. High genetic polymorphism from the cpSSR region suggests that cpSSR could be an effective tool for genetic diversity assessment and identification of Zantedeschia varieties. 


\section{Comparative analysis of four Zantedeschia chloroplast}

\section{2 genomes: expansion and contraction of the IR region,}

3 phylogenetic analyses and SSR genetic diversity assessment

4 Shuilian He Equal first author, 1, Yang Yang Equal first author, 2, Ziwei Li ${ }^{1}$, Xuejiao Wang', Yanbing

5 Guo ${ }^{1}$, Hongzhi Wu Corresp. 3

61 College of Horticulture and Landscape, Yunnan Agricultural University, Kunming, Yunnan, 7 650201, China;

82 College of Science, Yunnan Agricultural University, Kunming, Yunnan, 650201, China;

93 College of horticulture and landscape, Yunnan Agricultural University, Kunming, Yunnan, 10 China

11 Corresponding Author: Hongzhi Wu

12 Email address: 1994061@ynau.edu.cn

\section{ABSTRACT}

The horticulturally important genus Zantedeschia (Araceae) comprises eight species of

herbaceous perennials. We sequenced, assembled and analyzed the chloroplast (cp) genomes of four species of Zantedeschia (Z. aethiopica, Z. odorata, Z. elliottiana, and Z. rehmannii) to investigate the structure of the cp genome in the genus. According to our results, the cp genome of Zantedeschia ranges in size from 169,065 bp (Z. aethiopica) to 175,906 bp (Z. elliottiana). We identified a total of 112 unique genes, including 78 protein-coding genes, 30 transfer RNA (tRNA) genes and four ribosomal RNA (rRNA) genes. Comparison of our results with cp genomes from other species in the Araceae suggests that the relatively large sizes of the Zantedeschia $\mathrm{cp}$ genomes may result from inverted repeats (IR) region expansion. The sampled Zantedeschia species formed a monophylogenetic clade in our phylogenetic analysis. Furthermore, the long single copy (LSC) and short single copy (SSC) regions in Zantedeschia are more divergent than the IR regions in the same genus, and non-coding regions showed generally higher divergence than coding regions. We identified a total of $410 \mathrm{cpSSR}$ sites from the four Zantedeschia species studied. Genetic diversity analyses based on four polymorphic SSR markers from 134 cultivars of Zantedeschia suggested that high genetic diversity $(I=0.934$; 
$30 N e=2.371)$ is present in the Zantedeschia cultivars. High genetic polymorphism from the cpSSR

31 region suggests that cpSSR could be an effective tool for genetic diversity assessment and

32 identification of Zantedeschia varieties.

33 Keywords: Zantedeschia; chloroplast genome; genome comparison; IR expansion; phylogenetic 34 analysis, SSR 


\section{INTRODUCTION}

The genus Zantedeschia Spreng. (Trib. Richardieae, Araceae) had originally an entirely northeastern and southern African distribution. However, following introduction to Europe as ornamental plants in the seventeenth century, various species became widely naturalized across Europe, America, Oceania and Asia (Cruzcastillo et al. 2001). Two sections, Zantedeschia and Aestivae, are currently recognized in the genus Zantedeschia. Species in section Zantedeschia, Z. aethiopica and Z. odorata (Singh et al. 1996), can be recognized by the rhizomatous tuber and white flowers. Species in section Aestivae, however, have colorful (not white) flowers and discoid tubers (Singh et al. 1996; Wright \& Burge 2000). Many attractive and colorful hybrids between species in section Aestivae have been artificially produced, the majority between $Z$. albomaculata, Z. elliotiana, Z. rehmannii and Z. pentlandii (Snijder et al. 2004a). Zantedeschia hybrids have subsequently become one of the most popular horticultural crops worldwide, in high demand as cut flowers, potted plants and flower baskets, as well as for use in flower beds. The genus Zantedeschia is also of horticultural interest, however, F1 hybrids between sections Zantedeschia and Aestivae are invariably albino.

Traditional and polyploidization breeding, as well as resistance to soft rot, have been the main focuses for previous research into the genus Zantedeschia (Snijder et al. 2004a; Snijder et al. 2004b; Wright \& Triggs 2009; Wright \& Burge 2000; Wright et al. 2002). Simple sequence repeats (SSRs), or microsatellites, are short tandem repeats of two to more nucleotides in DNA sequences. The number of repeats is highly variable, whereas the regions of DNA flanking SSRs are highly conserved (Davierwala et al. 2000; Gur-Arie et al. 2000). SSR markers are polymerase chain reaction (PCR) -based, abundant, codominant, highly reproducible, and are distributed evenly across eukaryotic genomes (Powell et al. 1996). SSRs are widely used molecular markers to study genetic diversity, population structure, genetic mapping, phylogenetic studies, cultivar identification and marker-assisted selection. (Potter et al. 2015). A total of 43 novel EST-derived simple sequence repeat (SSR) markers have been identified in Zantedeschia by Wei et al (Wei et al. 2012), however, apart from this, the genetics and genomics 
62 of the genus Zantedeschia, which are of great importance in plant breeding, have received little

63

64 research attention. We therefore recommend that further genomic resources from Zantedeschia should be developed as tools to assist molecular breeding research in this genus. Our study focuses on four species of Zantedeschia, two from section Zantedeschia and two from section Aestivae. The aim of the study was to sequence, assemble and analyze the cp genome in Zantedeschia, to investigate any common characteristics or differences between the studied species and also to develop SSR markers in the Zantedeschia cp genome.

Photosysnthetic fixation of carbon in plants takes place in the chloroplasts, and is a primary function of these organelles. Chloroplasts have their own genome, as do mitochondria and it has been suggested that they were originally free-living cyanobacterium-like cells engulfed by ancient eukaryotic cells in an endosymbiotic relationship (Raven \& Allen 2003). The cp genome is usually represented as a circular molecule, and has a conserved quadripartite structure comprising the small single copy (SSC) and large single copy (LSC) regions, separated by two copies of an inverted repeat (IR) region. Chloroplast genomes have a highly conserved gene content, and most land plants have a nearly collinear gene sequence (Jansen et al. 2005). Due to their lack of recombination, their compact size and their maternal inheritance (Birky 2001), cp genomes are considered to be useful DNA sequences for plant genetic diversity assessment, plant identification and phylogenetic studies.

We investigated the cp genomes from four species of Zantedeschia. Genomes were sequenced, assembled, annotated and mined for the presence of SSR markers using Illumina sequencing technology. We also made comparative sequence analysis studies of the cp sequences from our study species. These results are publicly available as a genetic resource for the study of Zantedeschia species, and it is our hope that they will provide a valuable resource for future genetic and phylogenetic studies into this important genus.

\section{MATERIALS \& METHODS}


89

90

91

92

93

94

95

96

97

98

104

105

106

107

108

109

110

111

112

113

Plant material of Z. aethiopica was collected from South Africa directly and has been planted in Kunming more than 30 years. Z.odorata, Z. elliottiana, and Z. rehmannii were collected from Netherlands. Total genomic DNA of the four species of Zantedeschia was extracted from the fresh leaves of tissue culture seedlings using a modified CTAB extraction protocol based on Doyle \& Doyle (1987). Sequencing of the genomic DNA was performed using an Illumina Hiseq2000 (Illumina, CA, USA). Low quality reads were filtered out before de novo assembly of the cp genomes, and the resulting clean reads were assembled using the GetOrganelle pipeline (https://github.com/Kinggerm/ GetOrganelle). A reference genome Colocasia esculenta (JN105689) was used to check the contigs, using BLAST (https://blast.ncbi.nlm.nih.gov/), and the aligned contigs were then oriented according to the reference genome.

\subsection{Gene Annotation and Sequence Analysis}

The CpGAVAS pipeline (Liu et al. 2012) was used to annotate the genome and start/stop codons and intron/exon boundaries were adjusted in Geneious 8.1 (Kearse et al. 2012). The tRNA was identified using tRNAscan-SE v2.0 (Lowe \& Chan 2016), and sequence data were subsequently deposited in GenBank. The online tool OGDraw v1.2 (http://ogdraw.mpimpgolm.mpg.de/, Lohse et al. 2007) was used to generate a physical map of the genome.

\subsection{Structure of Genome and Genome comparison}

Pairwise sequence alignments of cp genomes were performed in MUMer (Kurtz et al. 2004). The complete cp genomes of the four species were then compared using mVISTA (Mayor et al. 2000 ) with the shuffle-LAGAN model Codon usage bias (RSCU) was calculated using MEGA v7.0 (Kumar et al. 2008). Chloroplast genome sequences of the four species were aligned using MAFFT (Katoh \& Standley 2013) in Geneious 8.1(Kearse et al. 2012). Insertion/deletion polymorphisms (indels) were then identified using DnaSP version 5.1 with the cp genome of $Z$. aethiopica as a reference (Librado \& Rozas 2009). Single nucleotide polymorphisms (SNPs), 
114

115

116

117

118

119

120

121

122

123

124

125

126

127

128

129

130

131

132

133

134

135

136

137

138

defined as variations in a single nucleotide that occur at specific positions in the genome, were called using a custom Python script (https://www.biostars.org/p/119214/).

\subsection{Phylogenetic analysis}

Chloroplast genome sequences of 11 species from Araceae and an outgroup (Zea mays, Poaceae) were downloaded from GenBank and an alignment with the four Zantedeschia cp genome sequences from our study was built using MAFFT (Katoh \& Standley 2013) in Geneious 8.1(Kearse et al. 2012). In order to investigate the phylogenetic placement of the genus Zantedeschia within the Araceae, a maximum likelihood tree was reconstructed in RaxML version 8.2.11 (Stamatakis 2014). Tree robustness was assessed using 1000 replicates of rapid four bootstrapping with the GTR+GAMMA substitution model.

\subsection{Simple Sequence Repeats (SSRs)}

SSR markers present in the Zantedeschia cp genome were found using Phobos v3.3.12 (Leese et al. 2008) and SSRHunter (Li \& Wan 2005). Both these programs search for repeats using a recursive algorithm. We set the minimum number of repeats of mono-, di-, tri-, tetra-, penta-, and hexa-nucleutide repeats to $10,5,4,3,3$ and 3 respectively. The inverted repeat region IRa was not considered in our SSR analysis.

We subsequently selected four SSRs motifs to investigate genetic diversity in Zantedeschia. A total of 134 cultivars from genus Zantedeschia were sampled. The experimented 134 cultivars include some local cultivars, but most of them are collected from Netherlands, the United States, New Zealand, and Taiwan for production and refreshed by Tissue culture every 3 years. Genetic diversity was investigated by calculating several indices: the number of alleles per locus $(\mathrm{Na})$; the number of fffective alleles $(\mathrm{Ne})$; Shannon's information index $(I)$ and polymorphism information content (PIC). Na, Ne, I were calculated using GenALEx v. 6.4 (Peakall \& Smouse 2006). PIC was calculated using PowerMarker 3.25 (Liu \& Muse 2006).

\section{RESULTS}


139

140

141

142

143

144

145

146

147

148

149

150

151

152

153

154

155

156

157

158

159

160

161

162

163

164

165

\subsection{Characteristics of Zantedeschia cp genomes}

After assembly and annotation, the four Zantedeschia cp genomes obtained in this study were submitted to the NCBI database (accession numbers MH743153-5 and MG432242). The cp genomes of these Zantedeschia species ranged in length from 169,065 bp (Z. aethiopica) to $175,906 \mathrm{bp}$ (Z. elliottiana), and, as expected, the cp genomes of all four species were found to contain both the large and small single-copy regions, separated by a pair of inverted repeat regions (Figure 1\& S1, Table 1). A total of 139 genes, of which 112 were unique, were identified, including 93 (78 unique) protein-coding genes, 38 (30 unique) transfer RNA (tRNA) genes and eight (four unique) ribosomal RNA (rRNA) genes (Table 2).

Interestingly, although the four species belong to a single genus, differences in gene content can nevertheless be seen. Z. elliottiana had the largest number of genes (139). Z. rehmannii had 138 genes, differing from Z. elliottiana only in a single copy of rps19. Z. odorata had 134 genes, and differs from Z. elliottiana in having only single copies of $n d h E, n d h G, r p s 19$, trnH-GUG and $\operatorname{trn} V-U A C$. Of all the species we studied, the cp genome of Z. aethiopica had the fewest of genes (131), and differed from Z. elliottiana in single copies of $n d h A, n d h E, n d h G, n d h H, n d h I, r p s 19$, $\operatorname{trn} H-G U G$ and trnV-UAC (Table 2).

The two species from section Aestivae (Z. elliottiana; Z. rehmannii) have larger cp genomes that those in section Zantedeschia (Z. aethiopica and Z. odorata), and the number of different protein-coding genes and tRNA genes is also higher in the $\mathrm{cp}$ genomes from plants in section Aestivae. Moreover, the size of the IR regions in species from section Aestivae was also larger than those in section Zantedeschia. Unlike the other studied species, which had no pseudogene, $Z$. odorata had two copies of the pseudogene $\Psi y c f 68$.

The nucleotide composition of the Zantedeschia cp genome was asymmetric, with an overall GC content ranging from $35.3 \%$ to $35.6 \%$, which is similar to other species in the Araceae (Tian et al. 2018). The largest GC content ratio was observed in the IR region (37.5\%$39.0 \%$ ), and the smallest in the SSC region (28.2\%-29.6\%). All four of our study species showed the same trend (the GC content of the LSC and SSC regions was lower than that of the IR 
166

167

168

169

170

171

172

173

174

175

176

177

178

179

180

181

182

183

184

185

186

187

188

189

190

191

192

regions), which may be due to the tRNA genes and rRNA genes generally having fewer AT nucleotides (Chen et al. 2015; Meng et al. 2018; Zhou et al. 2017).

Introns are non-coding sequences within genes, and they play a very important role in the regulation of gene expression (Jiang et al. 2017). Introns are known to accumulate more mutations than functional genes, and because of this are used extensively in phylogenetic and population genetics studies (Xu 2003). We investigated 13 intron-containing genes in the species Z. aethiopica: 11 genes ( $a t p F, n d h A, n d h B$, petB, petD, rpl16, rpl2, rpoC1, rps16, rps18, ycf68) contained only one intron, while two genes $(c l p P, y c f 3)$ contained two introns. Z. elliottiana and Z. rehmannii each had 12 intron-containing genes (similar to Z. aethiopica but lacking $c l p P$ ), and 11 intron-containing genes were found in Z. odorata (as Z. aethiopica but lacking rps 18 and ycf68) (Table S1).

\subsection{Genome comparison}

An extremely important topic in genomics is the comparative analysis of cp genomes (Chen et al. 2012; Zhihai et al. 2016). We performed multiple alignments between the four cp genomes generated in this study to characterize their divergence. The alignments were conducted in mVISTA, using $Z$. odorata as a reference (Figure 2).

Unsurprisingly, we found that in our study species, the coding regions are more conserved than the non-coding regions. Furthermore, the LSC and SSC regions are more divergent from each other than are the IR regions. Intergenetic spacers (including trnH-psbA, trnK-rps 16, rps 16$p s b K, \operatorname{trnT-trnL}, r b c L-p s a L, c l p P-p s b B, y c f 1-t r n L, \operatorname{trnL}-n d h B$ in the LSC regions and $p s a C-n d h E$, rps15-ycf1, trnL-ycf2 in the IR regions) were found to be the most divergent regions of the four $\mathrm{cp}$ genomes. Of the coding regions, the greatest divergence was found in $\operatorname{clp} P, r p l 16, r p s 19, y c f 1$ and $y c f 2$. This is in agreement with the results from previous studies (Park et al. 2017; Shen et al. 2017; Wu et al. 2017), and suggests that these regions might evolve rapidly in the genus

\section{Zantedeschia.}

The level of sequence divergence in the aligned cp genome sequences from our four study species was explored using nucleotide variability $(\pi)$, calculated using DnaSP version 5.1. The 
193

194

195

196

197

198

199

200

201

202

203

204

205

206

207

208

209

210

211

212

213

214

215

216

217

218

nucleotide variability $(\pi)$ of these sequences was 0.0487 , suggesting that the divergence between the cp genomes of these closely related species was relatively large. A total of 12,958 SNPs (including indels) were found. We infer from these results that the Zantedeschia cp genome could be suitable for species-level phylogenetic analyses.

\subsection{IR contraction and expansion in the Zantedeschia cp genome}

A detailed comparison of the IRs of Zantedeschia cp genome was conducted and is presented in Figure 3. The cp genome sequences of 11 other species of Araceae downloaded from NCBI were included in our analysis in order to investigate changes in the IR sequence in Zantedeschia.

The IR regions of Z. aethiopica, Z. odorata, Z. elliottiana and Z. rehmannii had lengths of $32,331 \mathrm{bp}, 36,549 \mathrm{bp}, 39,445 \mathrm{bp}$, and 38,354 bp, respectively. The even the shortest IR region of the four study species, that of Z. aethiopica, was longer than any of those from other species in the Araceae included in our study: Colocasia esculenta (25,273bp), Pinellia ternata $(25,625 \mathrm{bp})$, and Dieffenbachia seguine (25,235bp) (Tian et al. 2018). This expansion of the IR in the $Z$. aethiopica cp genome is because in this species, the rps15 gene has shifted from the SSC region to IRb at the SSC/IRb border, as well as to IRa at the SSC/Ira border. Other unusual, large expansions at the borders of IR regions have also been observed in our other three study species of Zantedeschia. In the two species Z. elliottiana and Z. rehmannii, the SSC/IRb border of occurs beside the $n d h E$ gene, meaning that six genes ( $n d h E, n d h G, n d h I, n d h A, n d h H, r p s 15)$ have shifted from the SSC region to the IR region in these species. In the case of $Z$. odorata, the $\mathrm{SSC} / \mathrm{IRb}$ border occurs beside the $n d h I$ gene, and four genes ( $n d h I, n d h A, n d h H$, and $r p s 15$ ) have therefore shifted from the SSC to the IR region. In all cases these shifts have resulted in a large expansion of the IR region.

\subsection{Phylogenetic analysis}

The cp genome sequences of 12 species (11 from the Araceae and the outgroup, Zea mays) were downloaded from NCBI, and the sequences were aligned together with the four 
219

220

221

222

223

224

225

226

227

228

229

230

231

232

233

234

235

236

237

238

239

240

241

242

243

244

245

Zantedeschia cp sequences from this study using Geneious 8.1 (Kearse et al. 2012). This alignment of the concatenated nucleotide sequences of a total of $16 \mathrm{cp}$ genome sequences (an ingroup of 15 species from Araceae and the ougroup, Zea mays) was subjected to phylogenetic analyses. The phylogeny was reconstructed using maximum likelihood (ML), and the resulting phylogenetic tree was found to be in agreement with the traditional genus-level morphological taxonomy of the Araceae (Figure 4). Furthermore, the topology is consistent with the classical taxonomy of Zantedeschia at the genus level (Singh et al. 1996). Our four study species from the genus Zantedeschia formed a monophyletic clade with 100\% bootstrap support. The morphologically defined sections Zantedeschia and Aestivae are also supported, with the two species from section Zantedeschia (Z. odorata and Z. aethiopica) sharing a more recent ancestor, and this clade forming a sister to the two species (Z. elliottiana, Z. rehmannii) from section Aestivae. This is the first time the cp genomes of these four Zantedeschia species have been sequenced, and the sequences have enriched the phylogenetic research in Araceae and we believe that they will provide a useful resource for the further study of the genetic diversity in this family.

\subsection{Simple Sequence Repeats (SSRs)}

The SSR survey of the four Zantedeschia species in this study identified 73, 107, 110, and 120 potential SSRs motifs in the cp genome sequences of Z. odorata (175,906 bp), Z. elliottiana (175,067 bp); Z. aethiopica (169,065 bp), and Z. rehmannii (173,783 bp), respectively. The observed frequency of SSRs motifs was therefore approximately one SSR motif per 1,400-2,500 bp of cp genome (Table S2). The majority of identified SSRs were mononucleotide repeats $(Z$. elliottiana: 52; Z. rehmannii: 55; Z. aethiopica: 61 ; Z. odorata: 54$)$, followed by dinucleotide repeats (Z. elliottiana: 32; Z. rehmannii: 31; Z. aethiopica: 20; Z. odorata: 14). Most SSR repeats were AT-rich, and only 38 SSR repeats in Zantedeschia contained cytosine. These results are consistent with previous findings that chloroplast SSRs usually consist of short polyA/T repeats (Nguyen et al. 2015). Most SSRs motifs were located in non-coding regions, in particular in the LSC region $(70.0 \%)$, or in the IR regions $(22.2 \%)$. Very few SSRs were located in the SSC 
246 region, and the ratio was less than $0.08 \%$. A similar result has been observed in other studies, 247 suggesting that the cp genome LSC region always contains high ratio of SSR motifs (Chi et al. 248 2018; Jian et al. 2018).

249 Four tri-SSRs motifs (Table 3) were used to investigate genetic diversity in Zantedeschia.

250 We sampled a total of 134 cultivars. All four cp SSR loci studied were polymorphic in the genus 251 Zantedeschia. The number of alleles $(\mathrm{Na})$ of the genus was 3.000, the number of effective alleles

$252(\mathrm{Ne})$ was 2.371, the Shannon's information index ( $I$ ) was 0.934 and the polymorphism 253 information content (PIC) was 0.388 (Table 4). These results suggest that chloroplast SSR 254 markers could be useful tools to study genetic diversity in Zantedeschia, and furthermore that 255 this could be an effective method to select germplasm for the improvement of ornamental 256 cultivars in Zantedeschia.

\section{DISCUSSION}

258

259

260

261

262

263

264

265

266

267

268

269

270

271

\section{IR contraction and expansion in the cp genome of the genus Zantedeschia}

With the exception of certain plants in the Fabaceae, and all conifers, the cp genomes of most plants display large inverted repeats (Aii et al. 1997). It has been suggested that these IRs have important roles in conserving essential genes and stabilizing the structure of chloroplast DNA (Palmer \& Thompson 1982). Most plant species have an IR of about $25 \mathrm{kbp}$ in size (Aii et al. 1997), and while the sequences of IRs are generally conserved, contraction and expansion events at the borders of these regions are common. During land plant evolution, there have been multiple instances of IR expansion or contraction that have involved the shifting of complete genes from the SSC regions into the IR or vice versa, resulting in the IR in land plants varying in size from 10 to $76 \mathrm{kbp}$. These events change the boundaries of the IR regions with the LSC or SSC regions, explaining the variation in size of the cp genome (Raubeson et al. 2007; Xia et al. 2009; Yao et al. 2015). The terminal IR gene, which is adjacent to the SSC region, is highly conserved across most land plants, and in most species, including those in the genera Rosa, Lancea and Paeonia (Meng et al. 2018), trnN-GUU is the last full-length IR gene at the IR/SSC 
272 boundary. This is strong evidence that this was an ancestral IR/SSC endpoint that has been 273 retained in most lineages (Zhu et al. 2016).

274 When we compared the cp genome IR boundary in various genera in the Araceae (Lemna, 275 Symplocarpus, Wolffia, Acorus, Symplocarpus, Pinellia, Colocasia and Dieffenbachia), we 276 found in this family, the IR generally terminates at the trnN-GUU gene at the IR/SSC boundary 277 (Figure 3) like most land plants. Howerver, we did find several minor IR extensions into the SSC 278 in the Araceae genera Acorus, Wolffiella, Spirodela, Lemna as well as in Z. aethiopica. Minor IR 279 extensions into the LSC region have occasionally occurred, as in Acorus americanus.

280 Surprisingly, large expansions have occurred in three species of Zantedeschia, and in particular in Z. elliottiana, in which six genes of the SSC region and two genes of the LSC region have

282 shifted into the IR region. The variation in the size of the IR region may not only explain the differences in length between different Zantedeschia $\mathrm{cp}$ genomes, but may also affect the rates of substitution and of plastome sequence evolution. Indeed, there is evidence that the IR has significant influence on the rates of evolution of plastid genomes, and the IR has been demonstrated to have lower rates of substitution (non-coding as well as synonymous and nonsynonymous) than do single-copy genes in several groups of angiosperms including carnivorous plants (Kim et al. 2009; Susann et al. 2013; Wolfe et al. 1987; Yi et al. 2012) as well as in some gymnosperms, such as Cycas (Wu \& Chaw 2015). However, in plants totally lacking the IR, such as the legume clade, those genes which in other groups are IR genes have a synonymous substitution rate similar to that of single-copy genes (Perry \& Wolfe 2002). Wolfe (1987) and Perry \& Wolfe (2002) suggest that a copy-dependent repair mechanism, such as gene conversion, would explain the lower rate of substitutions seen in the IR. Gene conversion has been demonstrated in plastids (Khakhlova \& Bock 2006), and is suggested to have been responsible for small increases and decreases in the size of the IR region (Goulding et al. 1996).

\section{SSR genetic diversity in Zantedeschia}

Genetic diversity assessment is used to characterize germplasm and also has a role in 
298

299

300

301

302

303

304

305

306

307

308

309

310

311

312

313

314

315

316

317

318

319

320

321

322

323

324

conservation, allowing the identification of potential parents for breeding programs (Friedt et al. 2007). Genetic diversity in germplasm collections is commonly assessed through the use of molecular markers. Inter-sample sequence repeats (ISSRs), amplified fragment length polymorphisms (AFLPs), and random amplified polymorphic DNA (RAPD) markers have allowed the development of DNA fingerprinting for the identification of cultivars of Zantedeschia (Bo et al. 2012; Hamada \& Hagimori 1996), revealing levels of genetic variation (Zhang 2009; Zhen \& XU 2013). However, as well as being labor-intensive and having only unstable reproducibility, a major weakness of these molecular markers is that they are dominant markers, and cannot therefore distinguish heterozygous and homozygous genotypes (Tan et al. 2012). Simple sequence repeat (SSR) markers possess several advantages over the other molecular markers, including co-dominance, high polymorphism, and good reproducibility (Morgante et al. 2002). Furthermore, SSRs from chloroplast DNA are powerful tools in evolutionary and population genetics (Dong et al. 2013; Dong et al. 2016; Flannery et al. 2006; Suo et al. 2016) for the construction of linkage maps and to inform the breeding of crop plants (Powell et al. 1995; Xue et al. 2012), because they are uniparentally inherited and can be highly variable even intraspecifically.

Wei et al. (2012) developed 43 polymorphic SSRs loci from expressed sequence tags (ESTs) from white calla lily (section Zantedeschia). Moderately high levels of genetic diversity were reported from analyses of 24 wild or cultivated accessions of white calla lily. The observed/expected heterozygosity $\left(H_{O} / H_{E}\right)$ was $0.501 / 0.662$, respectively, and the mean number of alleles per locus ( $\mathrm{Na}$ ) was 5.23. The PIC was found to be 0.446 (Wei et al. 2012). In a subsequent study into the genetic diversity of the colored calla lily (section Aestivae) using 31 EST-SSRs, Wei et al. (2017) showed that: $N a=3.58 ; H_{O}=0.453 ; H_{E}=0.478, \mathrm{PIC}=0.26$ and $N e=2.18$. Although the two studies both used EST-SSRs, evaluation of the genetic diversity revealed slight differences.

Our present study is the first to develop and employ SSR markers from the cp genome of genus Zantedeschia. In order to utilize these markers for the identification of cultivars, we 
325

326

327

328

329

330

331

332

333

334

335

336

337

338

339

340

341

342

343

344

345

346

347

348

349

350

351

choose four representative polymorphic tri-SSR markers and used these to assess genetic diversity in 134 cultivars of Zantedeschia. Compared with EST-SSRs diversity analyses from previous studies, our results show a low level of genetic diversity in Zantedeschia, with $\mathrm{Na}=$ 3.00, $\mathrm{Ne}=2.371$, and PIC $=0.388$. Furthermore, cpSSRs showed lower diversity than the nSSRs. Similar results have been reported from other species using both types of SSR markers (Pakkad et al. 2008; Robledo-Arnuncio \& Gil 2005; Setsuko et al. 2007), and reflects the low substitution rate in plant cpDNA sequences compared with that in nDNA (Wolfe et al. 1987). SSRs from mitochondrial or cp genomes have been developed in many species and have been used for the analysis of genetic diversity (Song et al. 2014; Wheeler et al. 2014), however this study represents the first time to develop the chloroplast SSR markers in Araceae. SSRs developed from the Zantedeschia uniparentally inherited and non-recombinant cp genome also have the advantages of nuclear SSRs, and we believe that they will be useful for genetic analysis in this horticulturally important genus.

\section{CONCLUSIONS}

This study presents the sequenced cp genome sequences from four horticulturally important species of Zantedeschia (Araceae), a genus native to northeastern and southern Africa and now globally naturalized. The sequencing, assembly, annotation and comparative analyses revealed that the cp genome of Zantedeschia has a quadruple structure, with a gene order and GC content similar to those of typical angiosperm cp genomes. However, unusual IR expansion was found in this genus. SSR genetic diversity assessment showed that Zantedeschia has moderately highlevel diversity. Phylogenetic analysis showed that the sampled species of the genus Zantedeschia formed a monophyletic clade. These sequences will enable us to assess genome-wide mutational dynamics within the family Araceae, and moreover, will facilitate investigations into gene expression and genetic variation within these ornamental species.

\section{ACKNOWLEDGEMENTS}

We thank Dr. Andan Zhu and Dr. Shudong Zhang from the Kunming Institute of Botany, Chinese Academy of Sciences for their help in the revision of the manuscript. 
352

353

354

355

356

357

358

359

360

361

362

363

364

365

366

367

368

369

370

371

372

373

374

375

376

377

378

\section{ADDITIONAL INFORMATION AND DECLARATIONS}

Supplementary Materials: Supplementary materials are available online.

Author Contributions:

- Shuilian He and Hongzhi Wu conceived and designed the experiments, performed the experiments, analyzed the data, contributed reagents/materials/analysis tools, prepared figures and/or tables, authored or reviewed drafts of the paper, and approved the final draft.

- Ziwei Li, Yang Yang Yanbing Guo and Xuejiao Wang conceived and designed the experiments, contributed analysis tools, prepared figures and/or tables, authored or reviewed drafts of the paper, and approved the final draft.

\section{REFERENCES}

Aii J, Kishima Y, Mikami T, and Adachi T. 1997. Expansion of the IR in the chloroplast genomes of buckwheat species is due to incorporation of an SSC sequence that could be mediated by an inversion. Current Genetics 31:276-279.

Birky CW. 2001. The inheritance of genes in mitochondria and chloroplasts: laws, mechanisms, and models. Annual Review of Genetics 35:125.

Bo LU, Zheng YH, Peng F, Shu XC, and Chen XX. 2012. Optimization of RAPD reaction system by uniform design on Zantedeschia hybrida. Northern Horticulture:123-126.

Chen S, Xu J, Liu C, Zhu Y, Nelson DR, Zhou S, Li C, Wang L, Guo X, and Sun Y. 2012. Genome sequence of the model medicinal mushroom Ganoderma lucidum. Nature Communications 3:913.

Chen X, Li Q, Li Y, Qian J, and Han J. 2015. Chloroplast genome of Aconitum barbatum var. puberulum (Ranunculaceae) derived from CCS reads using the PacBio RS platform. Frontiers in Plant Science 6:42.

Chi XF, Wang JL, Gao QB, Zhang FQ, and Chen SL. 2018. The complete chloroplast genomes of two Lancea species with comparative analysis. Molecules 23:602.

Cruzcastillo JG, Mendozaramirez J, and Torreslima PA. 2001. Shade, fertilizers and a natural bioregulator to improve Zantedeschia growth in a Mexican tropical upland area. Journal 
of agriculture of the University of Puerto Rico 85:135-142.

380

381

382

383

384

385

386

387

388

389

390

391

392

393

394

395

396

397

398

399

400

401

402

403

404

405

Davierwala AP, Ramakrishna W, Ranjekar PK, and Gupta VS. 2000. Sequence variations at a complex microsatellite locus in rice and its conservation in cereals. Theoretical and Applied Genetics 101:1291-1298.

Dong W, Chao X, Tao C, Lin K, and Zhou S. 2013. Sequencing angiosperm plastid genomes made easy: A Complete set of universal primers and a case study on the phylogeny of Saxifragales. Genome Biology and Evolution 5:989.

Dong W, Xu C, Li D, Jin X, Li R, Lu Q, and Suo Z. 2016. Comparative analysis of the complete chloroplast genome sequences in psammophytic Haloxylon species (Amaranthaceae). Peerj 4:e2699.

Doyle JJ, and Doyle JL. 1987. A rapid DNA isolation procedure for small quantities of fresh leaf tissue. Phytochemical Bull 19:11-15.

Flannery ML, Mitchell FJG, Coyne S, Kavanagh TA, Burke JI, Salamin N, Dowding P, and Hodkinson TR. 2006. Plastid genome characterisation in Brassica and Brassicaceae using a new set of nine SSRs. Theoretical and Applied Genetics 113:1221-1231.

Friedt W, Snowdon R, Ordon F, and Ahlemeyer J. 2007. Plant Breeding: assessment of genetic diversity in crop plants and its exploitation in breeding. Progress in Botany: Springer, Berlin, Heidelberg, 151-178.

Goulding SE, Wolfe KH, Olmstead RG, and Morden CW. 1996. Ebb and flow of the chloroplast inverted repeat. Molecular \& General Genetics Mgg 252:195-206.

Gur-Arie R, Cohen CJ, Eitan Y, Shelef L, and Kashi Y. 2000. Simple sequence repeats in Escherichia coli: abundance, distribution, composition, and polymorphism. Genome Research 10:62-71.

Hamada K, and Hagimori M. 1996. RAPD-based method for cultivar-identification of calla lily (Zantedeschia spp.). Scientia Horticulturae 65:215-218.

Jansen RK, Raubeson LA, Boore JL, Depamphilis CW, Chumley TW, Haberle RC, Wyman SK, Alverson AJ, Peery R, and Herman SJ. 2005. Methods for obtaining and analyzing whole 
406

407

408

409

410

411

412

413

414

415

416

417

418

419

420

421

422

423

424

425

426

427

428

429

430

431

432

chloroplast genome sequences. Methods in Enzymology 395:348.

Jian HY, Zhang YH, Yan HJ, Qiu XQ, Wang QG, Li SB, and Zhang SD. 2018. The complete chloroplast genome of a key ancestor of modern roses, Rosa chinensis var. spontanea, and a comparison with congeneric species. Molecules 23:389.

Jiang X, Yang C, Baosheng L, Shuiming X, Qinggang Y, Rui B, He S, Linlin D, Xiwen L, and Jun Q. 2017. Panax ginseng genome examination for ginsenoside biosynthesis. Gigascience 6:1-15.

Katoh K, and Standley DM. 2013. MAFFT multiple sequence alignment software version 7: improvements in performance and usability. Molecular Biology and Evolution 30:772.

Kearse M, Moir R, Wilson A, Stones-Havas S, Cheung M, Sturrock S, Buxton S, Cooper A, Markowitz S, and Duran C. 2012. Geneious Basic: An integrated and extendable desktop software platform for the organization and analysis of sequence data. Bioinformatics 28: 1647 .

Khakhlova O, and Bock R. 2006. Elimination of deleterious mutations in plastid genomes by gene conversion. Plant Journal 46:85-94.

Kim YK, Park C-w, and Kim K-J. 2009. Complete chloroplast DNA sequence from a Korean endemic genus, Megaleranthis saniculifolia, and its evolutionary implications. Molecules and Cells 27:365-381.

Kumar S, Nei M, Dudley J, and Tamura K. 2008. MEGA: a biologist-centric software for evolutionary analysis of DNA and protein sequences. Briefings in Bioinformatics 9:299306.

Kurtz S, Phillippy A, Delcher AL, Smoot M, Shumway M, Antonescu C, and Salzberg SL. 2004. Versatile and open software for comparing large genomes. Genome Biology 5:R12.

Leese F, Mayer C, and Held C. 2008. Isolation of microsatellites from unknown genomes using known genomes as enrichment templates. Limnology \& Oceanography Methods 6:412426.

Li Q, and Wan JM. 2005. SSRHunter: development of a local searching software for SSR sites. 
Hereditas 27:808.

434

435

436

437

438

439

440

441

442

443

444

445

446

447

448

449

450

451

452

453

454

455

456

457

458

459

Librado P, and Rozas J. 2009. DnaSP v5: a software for comprehensive analysis of DNA polymorphism data. Bioinformatics 25:1451-1452.

Liu C, Shi L, Zhu Y, Chen H, Zhang J, Lin X, and Guan X. 2012. CpGAVAS, an integrated web server for the annotation, visualization, analysis, and GenBank submission of completely sequenced chloroplast genome sequences. BMC Genomics 13:715.

Liu K, and Muse SV. 2006. PowerMarker: an integrated analysis environment for genetic marker analysis. Bioinformatics 21:2128-2129.

Lohse M, Drechsel O, and Bock R. 2007. OrganellarGenomeDRAW (OGDRAW): a tool for the easy generation of high-quality custom graphical maps of plastid and mitochondrial genomes. Current Genetics 52:267-274.

Lowe TM, and Chan PP. 2016. tRNAscan-SE On-line: integrating search and context for analysis of transfer RNA genes. Nucleic Acids Research 44:W54-W57.

Mayor C, Brudno M, Schwartz JR, Poliakov A, Rubin EM, Frazer KA, Pachter LS, and Dubchak I. 2000. VISTA : visualizing global DNA sequence alignments of arbitrary length. Bioinformatics 16:1046-1047.

Meng J, Li XP, Li HT, Yang JB, Wang H, and He J. 2018. Comparative analysis of the complete chloroplast genomes of four Aconitum medicinal species. Molecules 23:1015.

Morgante M, Hanafey M, and Powell W. 2002. Microsatellites are preferentially associated with nonrepetitive DNA in plant genomes. Nature Genetics 30:194-200.

Nguyen PAT, Kim JS, and Kim JH. 2015. The complete chloroplast genome of colchicine plants (Colchicum autumnale L. and Gloriosa superba L.) and its application for identifying the genus. Planta 242:223-237.

Pakkad G, Ueno S, and Yoshimaru H. 2008. Genetic diversity and differentiation of Quercus semiserrata Roxb. in northern Thailand revealed by nuclear and chloroplast microsatellite markers. Forest Ecology and Management 255:1067-1077.

Palmer JD, and Thompson WF. 1982. Chloroplast DNA rearrangements are more frequent when 
460

461

462

463

464

465

466

467

468

469

470

471

472

473

474

475

476

477

478

479

480

481

482

483

484

485

486

a large inverted repeat sequence is lost. Cell 29:537-550.

Park I, Kim WJ, Yeo SM, Choi G, Kang YM, Piao R, and Moon BC. 2017. The complete chloroplast genome sequences of Fritillaria ussuriensis Maxim. and Fritillaria cirrhosa D. Don, and comparative analysis with other Fritillaria species. Molecules 22:982.

Peakall R, and Smouse PE. 2006. GENALEX 6: genetic analysis in Excel. Population genetic software for teaching and research. Molecular Ecology Notes 6:288-295.

Perry AS, and Wolfe KH. 2002. Nucleotide Substitution Rates in Legume Chloroplast DNA Depend on the Presence of the Inverted Repeat. Journal of Molecular Evolution 55:501508.

Potter KM, Hipkins VD, Mahalovich MF, and Means RE. 2015. Nuclear genetic variation across the range of Ponderosa pine (Pinus ponderosa): Phylogeographic, taxonomic and conservation implications. Tree Genetics \& Genomes 11:38.

Powell W, Morgante M, Andre C, Hanafey M, Vogel J, Tingey S, and Rafalski A. 1996. The comparison of RFLP, RAPD, AFLP and SSR (microsatellite) markers for germplasm analysis. Molecular Breeding 2:225-238.

Powell W, Morgante M, Mcdevitt R, Vendramin GG, and Rafalski JA. 1995. Polymorphic simple sequence repeat regions in chloroplast genomes: applications to the population genetics of pines. Proceedings of the National Academy of Sciences of the United States of America 92:7759.

Raubeson LA, Peery R, Chumley TW, Dziubek C, Fourcade HM, Boore JL, and Jansen RK. 2007. Comparative chloroplast genomics: analyses including new sequences from the angiosperms Nuphar advena and Ranunculus macranthus. BMC Genomics 8:174.

Raven JA, and Allen JF. 2003. Genomics and chloroplast evolution: what did cyanobacteria do for plants? Genome Biology 4:1-5.

Robledo-Arnuncio JJ, and Gil L. 2005. Patterns of pollen dispersal in a small population of Pinus sylvestris L. revealed by total-exclusion paternity analysis. Heredity 94:13-22.

Setsuko S, Ishida K, Ueno S, Tsumura Y, and Tomaru N. 2007. Population differentiation and 
487

488

489

490

491

492

493

494

495

496

497

498

499

500

501

502

503

504

505

506

507

508

509

510

511

512

513

gene flow within a metapopulation of a threatened tree, Magnolia stellata (Magnoliaceae). American Journal of Botany 94:128-136.

Shen XF, Wu ML, Liao BS, Liu ZX, Bai R, Xiao SM, Li XW, Zhang BL, Xu J, and Chen SL. 2017. Complete chloroplast genome sequence and phylogenetic analysis of the medicinal plant Artemisia annua. Molecules 22:1330.

Singh Y, Wyk AEV, and Baijnath H. 1996. Taxonomic notes on the genus Zantedeschia Spreng. (Araceae) in southern Africa. South African Journal of Botany 62:321-324.

Snijder RC, Cho H-R, Hendriks MM, Lindhout P, and van Tuyl JM. 2004a. Genetic variation in Zantedeschia spp.(Araceae) for resistance to soft rot caused by Erwinia carotovora subsp. carotovora. Euphytica 135:119-128.

Snijder RC, Lindhout P, and van Tuyl JM. 2004b. Genetic control of resistance to soft rot caused by Erwinia carotovora subsp. carotovora in Zantedeschia spp. (Araceae), section Aestivae. Euphytica 136:319-325.

Song SL, Lim PE, Phang SM, Lee WW, Dang DH, and Prathep A. 2014. Development of chloroplast simple sequence repeats (cpSSRs) for the intraspecific study of Gracilaria tenuistipitata (Gracilariales, Rhodophyta) from different populations. BMC Research Notes 7:77-77.

Stamatakis A. 2014. RAxML version 8: a tool for phylogenetic analysis and post-analysis of large phylogenies. Bioinformatics 30:1312.

Suo Z, Li WY, Jin XB, and Zhang HJ. 2016. A new nuclear DNA marker revealing both microsatellite variations and single nucleotide polymorphic loci: A case study on classification of cultivars in Lagerstroemia indica L. Journal of Microbial \& Biochemical Technology 8:266-271.

Susann W, Bastian S, W. dC, and Müller KF. 2013. Disproportional plastome-wide increase of substitution rates and relaxed purifying selection in genes of carnivorous Lentibulariaceae. Molecular Biology and Evolution:3.

Tan C, Wu Y, Anderson MP, Tauer C, and Samuels T. 2012. Development of simple sequence 
514

515

516

517

518

519

520

521

522

523

524

525

526

527

528

529

530

531

532

533

534

535

536

537

538

539

540

repeat markers for bermuda grass from its expressed sequence tag sequences and preexisting sorghum SSR markers. Molecular Breeding 29:23-30.

Tian N, Han LM, Chen C, and Wang ZZ. 2018. The complete chloroplast genome sequence of Epipremnum aureum and its comparative analysis among eight Araceae species. PloS one 13:e0192956.

Wei Z-Z, Luo L-B, Zhang H-L, Xiong M, Wang X, and Zhou D. 2012. Identification and characterization of 43 novel polymorphic EST-SSR markers for arum lily, Zantedeschia aethiopica (Araceae). American Journal of Botany 99:e493-e497.

Wei Z, Zhang H, Wang Y, Li Y, Xiong M, Wang X, and Zhou D. 2017. Assessing genetic diversity and population differentiation of colored calla lily (Zantedeschia Hybrid) for an efficient breeding program. Genes 8:168.

Wheeler GL, Dorman HE, Alenda B, Lavanya C, and Wallace LE. 2014. A review of the prevalence, utility, and caveats of using chloroplast simple sequence repeats for studies of plant biology. Applications in Plant Sciences 2:1400059.

Wolfe KH, Li WH, and Sharp PM. 1987. Rates of nucleotide substitution vary greatly among plant mitochondrial, chloroplast, and nuclear DNAs. Proceedings of the National Academy of Sciences of the United States of America 84:9054-9058.

Wright P, and Triggs C. 2009. Factors affecting bacterial soft rot of Zantedeschia tubers. New Zealand Journal of Crop and Horticultural Science 37:345-350.

Wright PJ, and Burge GK. 2000. Irrigation, sawdust mulch, and Enhance ${ }^{\circledR}$ biocide affects soft rot incidence, and flower and tuber production of calla. New Zealand Journal of Crop and Horticultural Science 28:225-231.

Wright PJ, Burge GK, and Triggs CM. 2002. Effects of cessation of irrigation and time of lifting of tubers on bacterial soft rot of calla (Zantedeschia spp.) tubers. New Zealand Journal of Crop and Horticultural Science 30:265-272.

Wu CS, and Chaw SM. 2015. Evolutionary stasis in cycad plastomes and the first case of plastome GC-biased gene conversion. Genome Biology and Evolution 7:2000-2009. 
541 Wu ML, Li Q, Hu ZG, Li XW, and Chen SL. 2017. The complete Amomum kravanh chloroplast 542 genome sequence and phylogenetic analysis of the Commelinids. Molecules 22:1875.

543 Xia Z, Wang YZ, and Smith JF. 2009. Familial placement and relations of Rehmannia and Triaenophora (Scrophulariaceae s.1.) inferred from five gene regions. American Journal of Botany 96:519-530.

546

547

548

Xu JW. 2003. The first intron of rice EPSP synthase enhances expression of foreign gene. Science in China Ser C 46:561.

Xue J, Wang S, and Zhou SI. 2012. Polymorphic chloroplast microsatellite loci in Nelumbo (Nelumbonaceae). American Journal of Botany 99:240-244.

Yao X, Tang P, Li Z, Li D, Liu Y, and Huang H. 2015. The first complete chloroplast genome sequences in Actinidiaceae: Genome structure and comparative analysis. PloS one 10:e0129347.

Yi DK, Lee H-L, Sun B-Y, Chung MY, and Kim K-J. 2012. The complete chloroplast DNA sequence of Eleutherococcus senticosus(Araliaceae); Comparative evolutionary analyses with other three asterids. Molecules and Cells 33:497-508.

Zhang Y. 2009. Optimization of ISSR reaction system and preliminary study on Zantedeschia. Molecular Plant Breeding:827-832.

Zhen C, and XU. 2013. Physiological and biochemical and resistance changes and Issr polymorphic analysis exposed to $\sim(12) \mathrm{C} \sim(6+)$ heavy ion radiation on calla lily. Journal of Nuclear Agricultural Sciences 27:552-556.

Zhihai H, Jiang X, Shuiming X, Baosheng L, Yuan G, Chaochao Z, Xiaohui Q, Wen X, and Shilin C. 2016. Comparative optical genome analysis of two pangolin species: Manis pentadactyla and Manis javanica. Gigascience 5:1-5.

Zhou J, Chen X, Cui Y, Sun W, Li Y, Wang Y, Song J, and Yao H. 2017. Molecular structure and phylogenetic analyses of complete chloroplast genomes of two Aristolochia medicinal species. International Journal of Molecular Sciences 18:1839.

Zhu A, Guo W, Gupta S, Fan W, and Mower JP. 2016. Evolutionary dynamics of the plastid 
568 inverted repeat: the effects of expansion, contraction, and loss on substitution rates. New Phytologist 209:1747-1756.

570

571

572 
Figure 1

Gene map of chloroplast genome of $Z$. odorata .

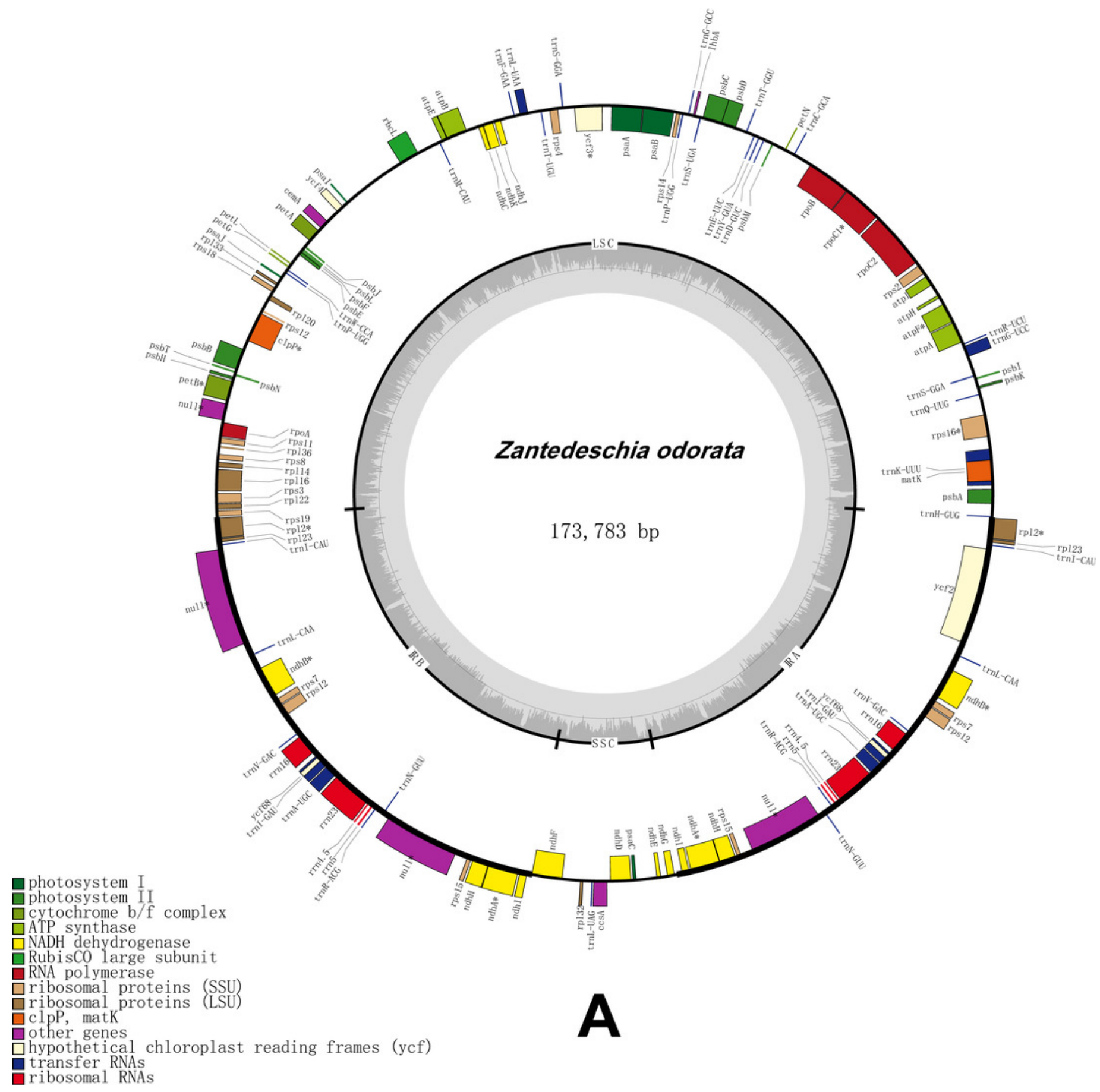




\section{Figure 2}

Comparison of four $\mathrm{cp}$ genomes using the mVISTA alignment program.

The $x$-axis represents the coordinates in the $c p$ genome. The $y$-axis indicated the average percent identity of sequence similarity in the aligned regions, ranging between $50 \%$ and $100 \%$, Purple bars represent exons, blue bars represent untranslated regions (UTRs), pink bars represent noncoding sequences (CNS), gray bars represent mRNA, and white bars represent differences of genomics. 


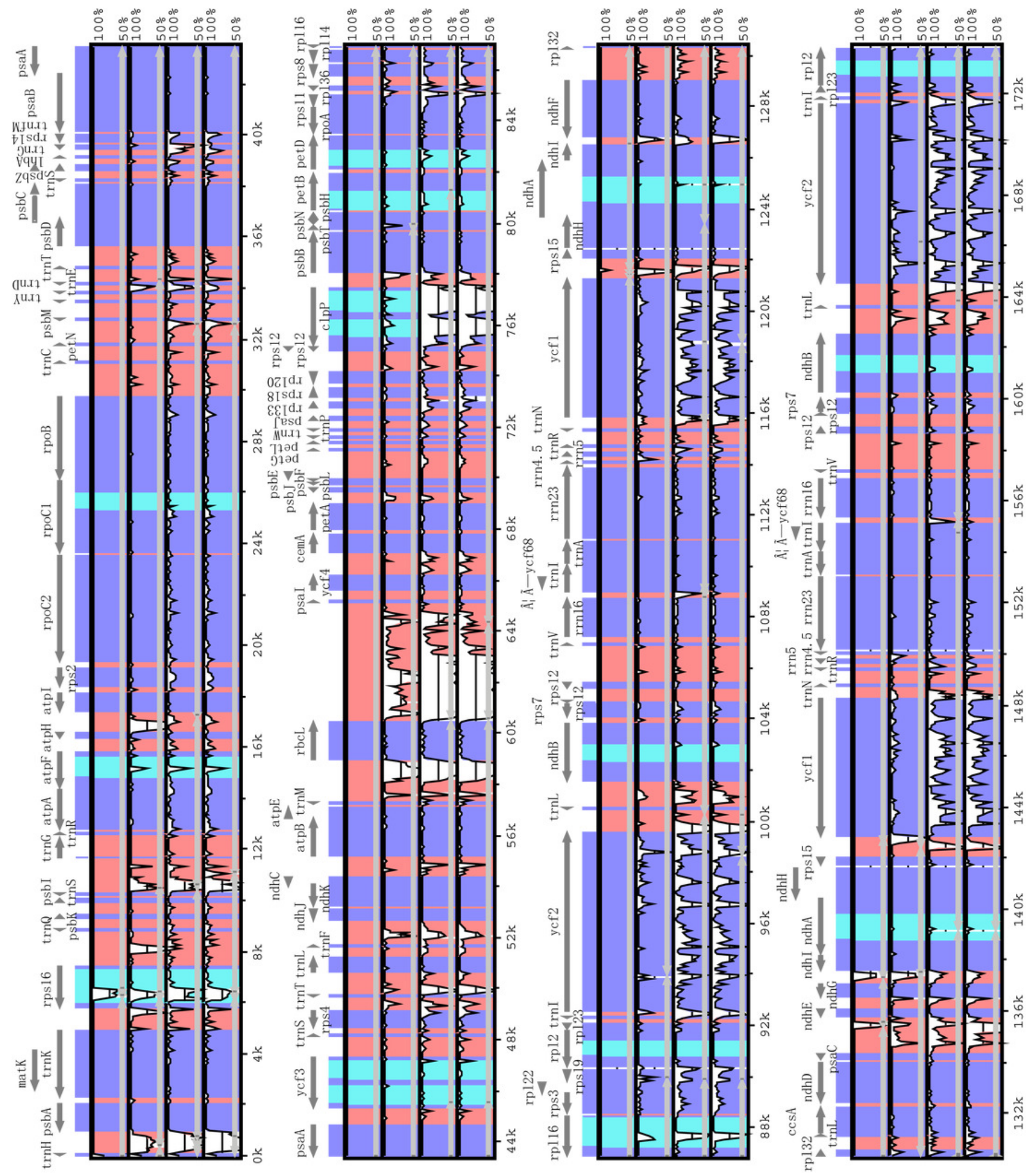

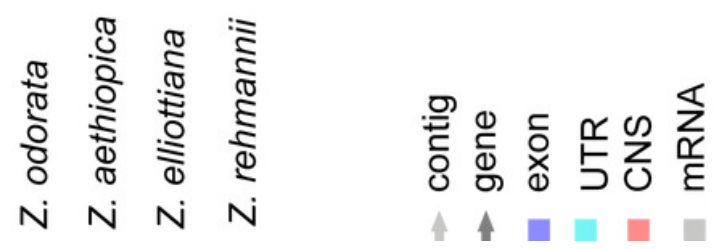


Figure 3

\section{Genes of IR region in Araceae (Genes which are only partially duplicated in the IR are not shown).}

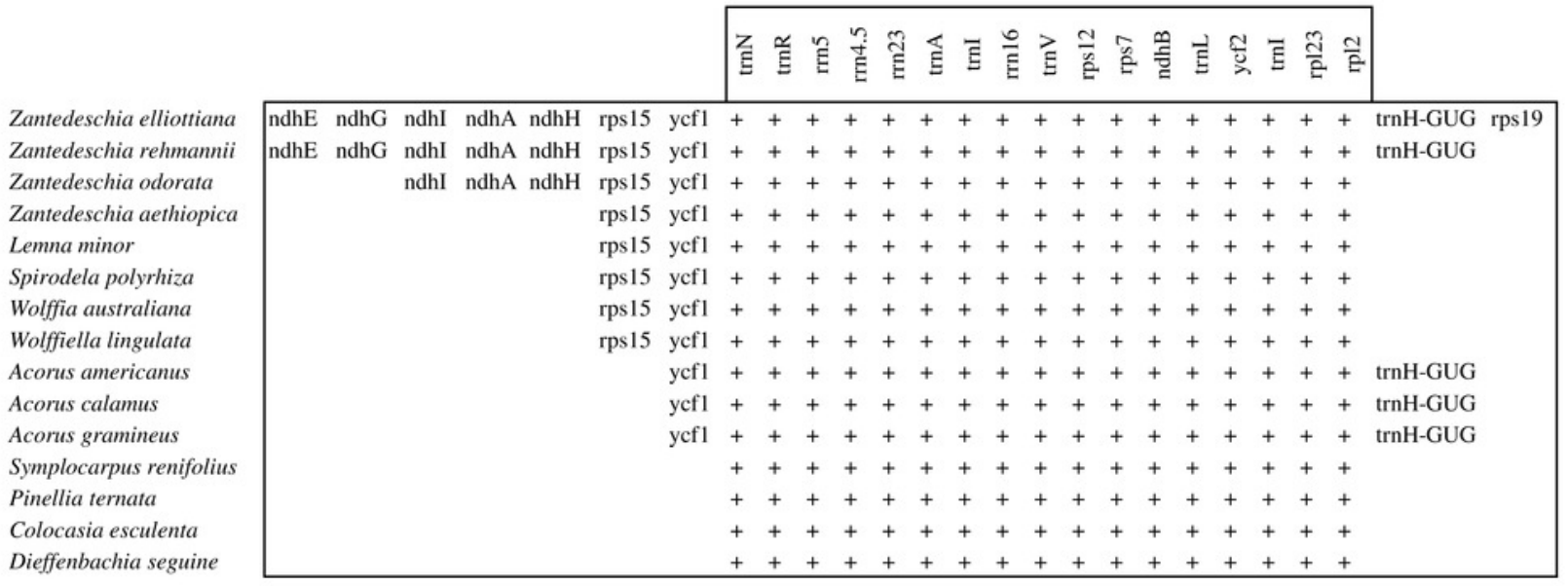


Figure 4

The maximum likelihood (ML) phylogenetic tree based on 14 complete chloroplast genome sequence.

Numbers at the right of nodes are bootstrap support values. 


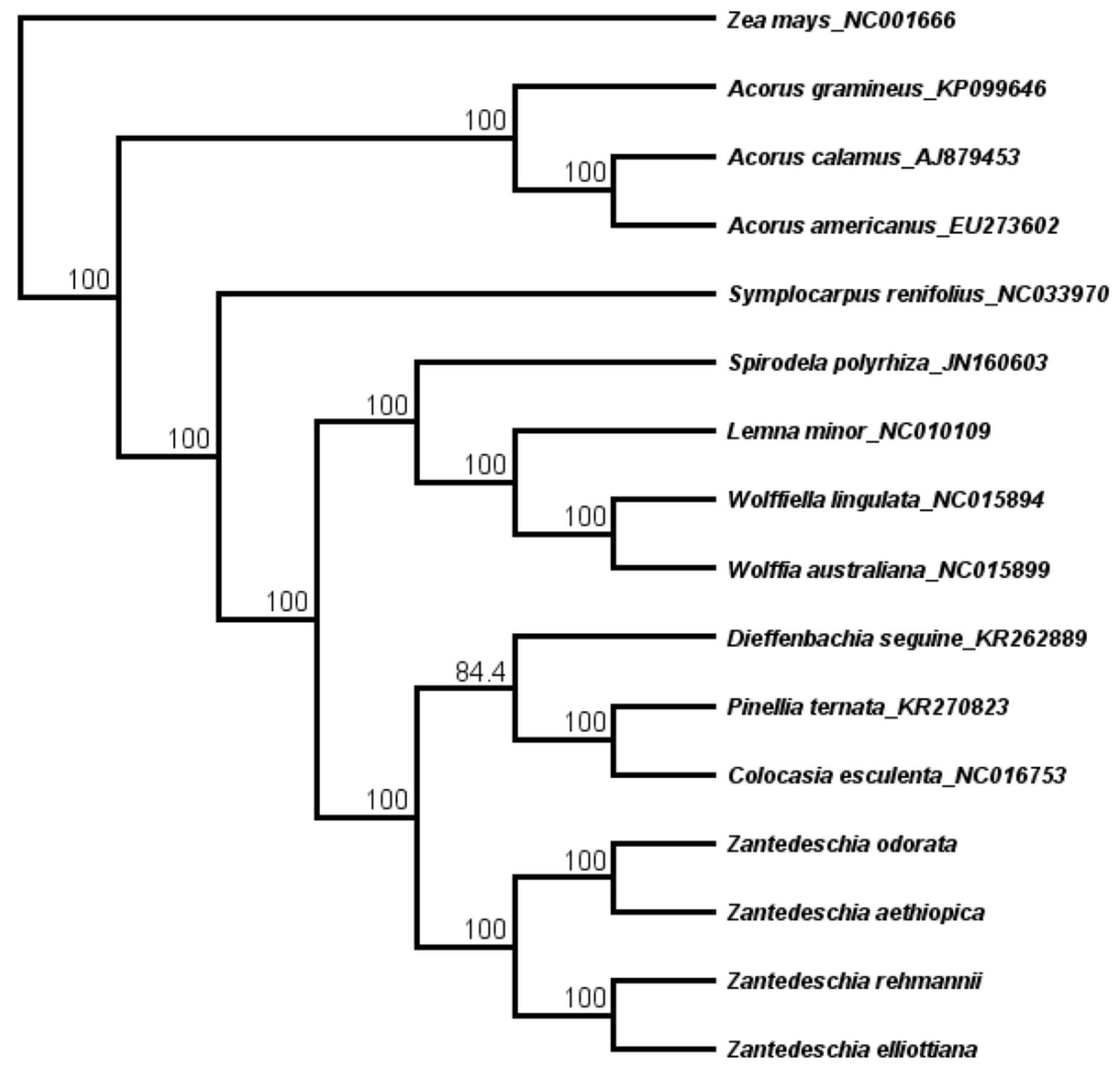




\section{Table $\mathbf{1}$ (on next page)}

The basic characteristics of chloroplast genomes of four Zantedeschia species 
1 Table 1. The basic characteristics of chloroplast genomes of four Zantedeschia species.

\begin{tabular}{lllll}
\hline Characteristics & Z. elliottiana & Z. rehmannii & Z. aethiopica & Z. odorata \\
\hline GenBank numbers & MH743153 & MH743154 & MH743155 & MG432242 \\
Total cp genome size/bp & 175,906 & 175,067 & 169,065 & 173,783 \\
LSC size/bp & 88,584 & 90,020 & 89,695 & 90,322 \\
IR size /bp & 39,445 & 38,354 & 32,331 & 36,549 \\
SSC size /bp & 8,432 & 8,338 & 14,715 & 10,363 \\
Total number of genes & 139 & 138 & 131 & 134 \\
Number of different protein-coding & 93 & 92 & 87 & 90 \\
genes & 38 & 38 & 37 & 36 \\
Number of different tRNA genes & 38 & 8 & 8 & 8 \\
Number of different rRNA genes & 8 & 52 & 40 & 46 \\
Number of gene in IR region & 54 & 0 & 0 & 2 \\
Number of pseudogene & 0 & 35.6 & 35.6 & 35.3 \\
GC content (\%) & 35.4 & 34.4 & 34.1 & 33.7 \\
GC content of LSC (\%) & 34.2 & 37.7 & 39.0 & 38.2 \\
GC content of IR (\%) & 37.5 & 29.1 & 29.6 & 28.2 \\
GC content of SSC (\%) & 28.7 & & & \\
\hline
\end{tabular}

2 


\section{Table 2 (on next page)}

Genes present in the Zantedeschia chloroplast genome

${ }^{2}$ Two gene copies in IRs. ${ }^{\%}$ shows only one copy in Z. rehmannii, ${ }^{\oplus}$ shows only one copy in Z. aethiopica, ${ }^{\circledR}$ shows only one copy in $Z$. odorata, ${ }^{*}$ shows gene not exist in $Z$. aethiopica, *shows gene not exist in Z. odorata. $\psi$ shows pseudogenes. 
1 Table 2. Genes present in the Zantedeschia chloroplast genome.

\begin{tabular}{|c|c|c|}
\hline Category & Gene groups & Name of genes \\
\hline \multirow[t]{5}{*}{ Self-replication } & $\begin{array}{l}\text { Large subunit of } \\
\text { ribosomal proteins }\end{array}$ & rpl2 ${ }^{2}, r p l 14, r p l 16, r p l 20, r p l 22, r p l 23^{2}$, rpl32, rpl33, rpl36 \\
\hline & $\begin{array}{l}\text { Small subunit of } \\
\text { ribosomal proteins }\end{array}$ & $\begin{array}{l}\text { rps2, rps3, rps4, rps } 7^{2}, r p s 8, r p s 11, r p s 12^{2}, r p s 14, r p s 15^{2}, r p s 16 \text {, } \\
\text { rps18, rps192@\&\% }\end{array}$ \\
\hline & $\begin{array}{l}\text { DNA dependent RNA } \\
\text { polymerase }\end{array}$ & гроA, гроB, гроC1, гроС2 \\
\hline & $\begin{array}{l}\text { Ribosomal RNA } \\
\text { genes }\end{array}$ & $r r n 4.5^{2}, r r n 5^{2}, r r n 16^{2}, r r n 23^{2}$ \\
\hline & Transfer RNA genes & $\begin{array}{l}\operatorname{trn} A(U G C)^{2}, \operatorname{trn} C(G C A), \operatorname{trn} D(G U C), \operatorname{trn} E(U U C), \operatorname{trn} F(G A A), \\
\operatorname{trn} F(C A U), \operatorname{trn} G(G C C), \operatorname{trn} G(U C C), \operatorname{trn} H(G U G)^{2 @ \&}, \\
\operatorname{trn} I(C A U)^{2}, \operatorname{trn} I(G A U)^{2}, \operatorname{trn} K(U U U), \operatorname{trn} L(C A A)^{2}, \operatorname{trn} L(U A A), \\
\operatorname{trn} L(U A G), \operatorname{trn} M(C A U), \operatorname{trn} N(G U U)^{2}, \operatorname{trn} P(U G G), \operatorname{trn} Q(U U G), \\
\operatorname{trn} R(A C G)^{2}, \operatorname{trn} R(U C U), \operatorname{trn} S(G C U), \operatorname{trn} S(G G A), \operatorname{trn} S(U G A), \\
\operatorname{trn} T(G G U), \operatorname{trn} T(U G U), \operatorname{trn} V(G A C)^{2}, \operatorname{trn} V(U A C)^{* \#,} \operatorname{trn} W(C C A), \\
\operatorname{trn} Y(G U A)\end{array}$ \\
\hline \multirow[t]{6}{*}{ Photosynthesis } & $\begin{array}{l}\text { NADH } \\
\text { oxidoreductase }\end{array}$ & $\begin{array}{l}n d h A^{2 @}, n d h B^{2}, n d h C, n d h D, n d h E^{2 @ \&}, n d h F, n d h G^{2 @ \&}, n d h H^{2 @}, \\
n d h I^{2 @}, n d h J, n d h K\end{array}$ \\
\hline & Photosystem I & psaA, psaB, psaC, psaI, psaJ,ycf3, ycf4 \\
\hline & Photosystem II & $\begin{array}{l}\text { psbA, psbB, psbC, psbD, psbE, psbF, psbH, psbI, psbJ, psbK, } \\
\text { psbL, psbM, psbN, psbT, psbZ }\end{array}$ \\
\hline & $\begin{array}{l}\text { Cytochrome b/f } \\
\text { complex }\end{array}$ & $\operatorname{pet} A, \operatorname{pet} B, \operatorname{pet} D, \operatorname{pet} G, \operatorname{pet} L, \operatorname{pet} N$ \\
\hline & ATP synthase & atpA, atpB, atpE, atpF, atpH, atpI \\
\hline & RubisCo large subunit & $r b c L$ \\
\hline \multirow[t]{5}{*}{ Other genes } & Maturase K & matK, cemA \\
\hline & $\begin{array}{l}\text { C-type cytochrome } \\
\text { synthesis gene }\end{array}$ & $\operatorname{ccs} A$ \\
\hline & Protease & $\operatorname{clpP}$ \\
\hline & $\begin{array}{l}\text { Proteins of unknown } \\
\text { function }\end{array}$ & $y c f 1^{2}, y c f 2^{2}, y c f 68^{2^{*}}$ \\
\hline & pseudogene & $\Psi y c f 68^{2}$ (in Z. odorata) \\
\hline
\end{tabular}

$2 \quad{ }^{2}$ Two gene copies in IRs. ${ }^{\%}$ shows only one copy in Z. rehmannii, ${ }^{\circledR}$ shows only one copy in Z. aethiopica, ${ }^{\circledR}$ shows 3 only one copy in Z. odorata, " shows gene not exist in Z. aethiopica, *shows gene not exist in Z. odorata. $\Psi$ shows 4 pseudogenes. 


\section{Table 3 (on next page)}

Characteristics of the four SSR motifs for $Z$. odorata. Forward and reverse primer sequences, Annealing temperature (Tm), repeat motifs. 
1 Table 3. Characteristics of the four SSR motifs for Z. odorata. Forward and reverse primer 2 sequences, Annealing temperature (Tm), repeat motifs.

\begin{tabular}{llllllll}
\hline Primer & repeat & Start(bp) & End(bp) & Forword Primer $\left(\mathbf{5}^{\prime}-\mathbf{3}^{\prime}\right)$ & $\mathbf{T m}\left({ }^{\circ} \mathbf{C}\right)$ & Reverse Primer $\left(\mathbf{5}^{\prime}-\mathbf{3}^{\prime}\right)$ & $\mathbf{T m}\left({ }^{\circ} \mathbf{C}\right)$ \\
\hline 1 & (A) 10 & 4957 & 4966 & CATAGCCGCACTTAAAAGCC & 59.875 & TGGGATCGTGCAATCAATTT & 61.239 \\
2 & (A) 10 & 12561 & 12570 & CCATAAAGGAGCCGAATGAA & 60.031 & AGACAATGGACGCTGCTTTT & 59.882 \\
3 & (A) 10 & 40167 & 40176 & ATCCCCTTCTCCATCGAAAT & 59.728 & AGCAAGATTGGTTGGATTGG & 59.933 \\
4 & (T) 10 & 76955 & 76964 & GGGCAAATTATGTCAGTGCC & 60.339 & AGGCTATCTCAAACTGCCGA & 59.978 \\
\hline
\end{tabular}

3 


\section{Table 4 (on next page)}

Genetic diversity parameters estimated on 134 Zantedeschia accessions

Note: $\mathrm{Na}=$ No. of Alleles $\mathrm{Na}($ Freq $>=5 \%)=$ No. of Different Alleles with a Frequency $>=5 \%$ Ne $=$ No. of Effective Alleles I = Shannon's Information Index 
1 Table 4. Genetic diversity parameters estimated on 134 Zantedeschia accessions

\begin{tabular}{llll}
\hline Parameter & Section Zantedeschia & Section Aestivae & total \\
\hline $\mathrm{Na}$ & 3.000 & 14.000 & 14.000 \\
$\mathrm{Na}$ Freq. $>=5 \%$ & 3.000 & 6.500 & 7.000 \\
No. Private Alleles & 0.000 & 11.000 & 14.000 \\
$\mathrm{Ne}$ & 2.295 & 6.084 & 6.307 \\
$\mathrm{I}$ & 0.844 & 2.116 & 2.130 \\
\hline
\end{tabular}

2 Note: $\mathrm{Na}=$ No. of Alleles

$3 \mathrm{Na}($ Freq $>=5 \%)=$ No. of Different Alleles with a Frequency $>=5 \%$

$4 \quad \mathrm{Ne}=$ No. of Effective Alleles

$5 \quad I=$ Shannon's Information Index

6

7 